\title{
How can we work our microbiome to improve our overall health and immunity - the good, the bad, and the ugly?
}

\author{
Frédéric Raymond, PhD 1,2 and Jacques Corbeil, PhD ${ }^{1,2,3}$
}

1. Centre de Recherche en Infectiologie, CHU de Québec Université Laval, Québec, Canada. 2. Centre de Recherche en Données Massives de I'Université Laval, Université Laval, Québec, Canada. 3. Département de Médecine Moléculaire, Université Laval, Québec, Canada.

Some see the microbiome as the new Wild West, a biological frontier to explore, populated with bacteria competing for ecological space. Studying the microbial populations within our bodies should lead to a better understanding of the role of microbes in our lives and, notably, the discovery of new ways to maintain and even improve our health. However, as in all good westerns, there is the good, the bad and the ugly. Now, let us explore the gut microbiome.

Most of the bacteria in our intestine benefit our organism and, as a whole, can even be considered as an organ. They are commensals and symbionts; we provide them with food and shelter while they perform essential functions like digestion and protection from pathogens. Due to its plasticity, our microbiome can evolve faster than our genome and therefore allow adaptation to external factors such as changes in food sources. For example, transfer of genes from marine bacteria to the gut microbiome of Japanese individuals could have allowed a better digestion of the seaweeds used in sushi (1).

Then there are the bad; pathogens or, more precisely, what some call the pathobionts (2). These are opportunistic pathogens that, in the right conditions, can cause infections. One of these turncoats is Clostridium difficile. This bacteria is present in $2-17 \%$ of healthy adults and lives a seemingly peaceful life inside a healthy intestine (3). However, when elderly people are exposed to antibiotics, the disturbance in their gut microbiome leads to an increase in C. difficile, which in turn can increase toxin production (4). These toxins are correlated with the severity of infection.

Finally, there's the ugly - not a specific microorganism but rather our lack of knowledge on the impact of external factors on the gut microbiome. We have known for years that antibiotics can alter the bacterial population within our gut, still very few studies have thoroughly evaluated the impact of antibiotic use on individuals. This is what we set out to do in our 2015 study which evaluated the impact of an antibiotic, Cefprozil, on the gut microbiome of healthy individuals (5). Previous research using this antibiotic suggested that it had a limited effect on the microbiome and few side effects (6). What we observed was somewhat different; indeed, the bacteria that comprised the majority of the gut microbiome were not affected by Cefprozil. Any impact was primarily observed in low abundance bacteria, whose precise functions are still not properly characterized. Only two bacteria were consistently increased after antibiotic treatment; one of them was Lachnoclostridium bolteae, a cousin of $C$. difficile. In addition, we observed a bloom of the opportunistic pathogen Enterobacter cloacae within a subset of individuals. These individuals also had lower initial microbiome diversity. This shows that antibiotics are not only modifying the gut microbiome, but that these modifications depend on the individual's initial microbiota.

This implies that medication and other medical interventions affect our microbiome in ways that we have yet to understand and predict. In the coming years we need to evaluate the effects of drugs on the microbiome in order to understand their hidden impact on their host. Only then will we be able to tame the ugly and guarantee that our microbiome remains beneficial to human health.

\section{References}

1. Hehemann JH, Correc G, Barbeyron T, Helbert W, Czjzek M, Michel G. Transfer of carbohydrate-active enzymes from marine bacteria to Japanese gut microbiota. Nature. 2010 Apr 8;464(7290):908-12.

2. Chow J, Tang H, Mazmanian SK. Pathobionts of the gastrointestinal microbiota and inflammatory disease. Curr Opin Immunol. 2011 Aug;23(4):473-80.

3. Rodriguez C, Taminiau B, Van Broeck J, Delmée M, Daube G. Clostridium difficile infection and intestinal microbiota interactions. Microb Pathog. 2015 Dec;89:201-9.

4. Martin JS, Monaghan TM, Wilcox MH. Clostridium difficile infection: epidemiology, diagnosis and understanding transmission. Nat Rev 
Gastroenterol Hepatol. 2016 Mar 9.

5. Raymond F, Ouameur AA, Déraspe $M$, Iqbal N, Gingras $H$, Dridi B, et al. The initial state of the human gut microbiome determines its reshaping by antibiotics. ISME J. 2016 Mar;10(3):707-720.

6. Edlund C, Nord C. Effect on the human normal microflora of oral antibiotics for treatment of urinary tract infections. I Antimicrob Chemother. 2000;46:41-48.

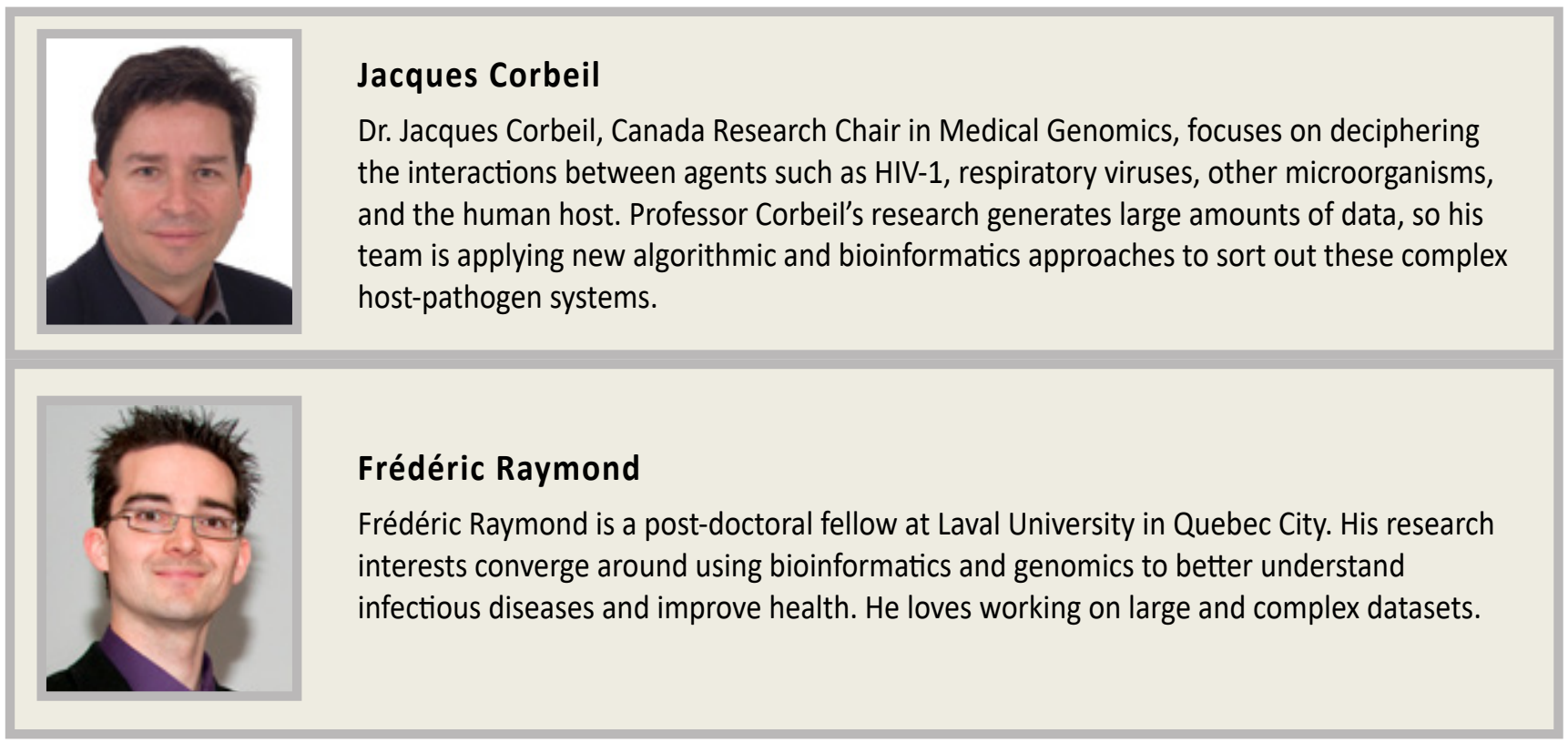

